\title{
The Crystallization and Properties of Sputter Deposited Lithium Niobite
}

\author{
Joshua C. Shank, M. Brooks Tellekamp, W. Alan Doolittle* \\ Department of Electrical and Computer Engineering, Georgia Institute of Technology, Atlanta, GA 30332 \\ *Please direct all correspondence to: Tel: +4048949884 or Email: alan.doolittle@ece.gatech.edu
}

\begin{abstract}
Sputter deposition of the thin film memristor material, lithium niobite $\left(\mathrm{LiNbO}_{2}\right)$ is performed by co-deposition from a lithium oxide $\left(\mathrm{Li}_{2} \mathrm{O}\right)$ and a niobium target. Crystalline films that are textured about the (101) orientation are produced under room temperature conditions. This material displays memristive hysteresis and exhibits XPS spectra similar to MBE and bulk grown $\mathrm{LiNbO}_{2}$. Various deposition parameters were investigated resulting in variations in the deposition rate, film crystallinity, oxygen to niobium ratio, and mean niobium oxidation state. The results of this study allow for the routine production of large area $\mathrm{LiNbO}_{2}$ films at low substrate temperature useful in hybrid-integration of memristor, optical, and energy storage applications.
\end{abstract}

\section{Keywords}

Lithium Niobite; Sputter Deposition; X-ray Diffraction, X-ray Photoelectron Spectroscopy

\section{Introduction}

Lithium niobite $\left(\mathrm{LiNbO}_{2}\right)$ is a sub-oxide of the more common acoustic and optical wave-guiding material lithium niobate $\left(\mathrm{LiNbO}_{3}\right)$. Lithium niobite is a mixed ion-electron conductor that has been explored for battery [1] and neuromorphic [2] applications. It is a layered [3] semiconductor [1] with loosely bound lithium ions [4, 5]. Deintercalation of the lithium ions, forming $\mathrm{Li}_{x} \mathrm{NbO}_{2}(0<x<1)$, yields a degenerately doped $p$-type $[6,7]$ material with a high density of states at the Fermi energy [7-9]. Modulation of the lithium content by chemical [1], electrical [10], or optical [11] means creates opportunities for multi-functional devices based on the resulting memristive $[12,13]$ and optical changes $[1,3,11]$. Additionally, a low temperature superconducting transition was found for lithium deintercalated material $\left(\mathrm{Li}_{\times} \mathrm{NbO}_{2}\right)$ [14] despite the band structure similarities to high temperature cuprate superconductors $[6,7]$.

Early work on lithium niobite was performed on powdered material that likely contained unreacted precursors [3] or other impurities [9] leading to discrepancies in experimental measurements such as both metallic [7] and semiconducting [1] temperature dependent resistivity as well as diamagnetic [4], paramagnetic [1, 14], and antiferromagnetic [3] behavior. Such discrepancies have been previously noted in the literature [7, 9]. Later work was performed on single crystal material in bulk form grown by liquid phase electro-epitaxy with a molten salt solution $[7,15]$ and in thin films grown by molecular beam epitaxy using a metal-chloride chemistry $[2,16]$. While both of these 
methods for growing single crystalline lithium niobite involve high temperature corrosive environments, herein we report a method for sputter deposition of lithium niobite using traditional vacuum technologies common in industrial microfabrication and facilitating integration with other semiconductor technologies for advanced functionality.

\section{Experimental Setup}

All sputter depositions reported herein were performed on a modified Denton Discovery 18 sputtering system with three confocal sputtering cathodes, an electrically isolated platen that can be heated above $800^{\circ} \mathrm{C}$, and controlled flows of $\mathrm{Ar}, \mathrm{O}_{2}$, and $\mathrm{N}_{2}$ process gases. The system was pumped to a base pressure of $3 \times 10^{-7}$ Torr using a turbo pump and a cryo pump. During deposition the pressure was controlled using a constant gas flow and a variable conduction throttle valve on the turbo pump. Argon was chosen for the working gas and no reactive species were introduced.

This work explores 1 hour co-depositions from an RF energized lithium oxide $\left(\mathrm{Li}_{2} \mathrm{O}\right)$ target and a DC energized niobium target on to c-plane sapphire substrates. The sputtering targets measure 3.625 inches in diameter and are indium bonded directly to the water cooling blocks for improved heat transfer. The platen was water cooled to remove excess heat produced by the sputtering process.

The physical properties of the deposited films were analyzed using x-ray diffraction (XRD) and cross sectional scanning electron microscopy (SEM). XRD was performed with a Panalytical $\mathrm{x}$-ray diffractometer using $\mathrm{Cu} \mathrm{K}$-alpha radiation. Cross sectional SEM was performed on a Hitachi SU8230 electron microscope to determine the film thickness. Cross sectional samples were prepared by tensile fracture.

XRD 20- $\omega$ scans were performed between $10-80$ degrees in order to determine the crystallinity of the deposited film and identify phases. The crystallinity of the film was determined from the XRD peak height scaled by the sapphire substrate peak height and the film thickness. All of the deposited films produced an XRD peak between $2 \theta-\omega=36.39^{\circ}-36.87^{\circ}$ corresponding to the XRD peak for the (101) orientation of $\mathrm{LiNbO}_{2}$. The location of this peak can vary due to strain and variations in lithium or oxygen content. Changes in lithium content, $\mathrm{Li}_{x} \mathrm{NbO}_{2}$ where $0.5<x<1$, change the hexagonal lattice spacing between $\mathrm{a}=2.900-2.925 \AA$ and $\mathrm{c}=10.46-$ $10.465 \AA$ [1]. This range of unstrained lattice constants results in a range of XRD angles between $2 \theta-\omega=36.48^{\circ}-36.77^{\circ}$.

In addition to variations in the (101) oriented $\mathrm{Li}_{x} \mathrm{NbO}_{2}$ XRD peak location, alternative phases with notable XRD peaks near this range make exact phase identification difficult via XRD alone. Other phases with XRD peaks near the measured peaks include $\mathrm{Nb}_{\mathrm{FCC}}(111)$ at $36.77^{\circ}$, $\mathrm{NbO}(111)$ at $36.95^{\circ}, \mathrm{LiNb}_{3} \mathrm{O}_{8}(600)$ at $36.99^{\circ}$, and $\mathrm{Li}_{3} \mathrm{NbO}_{4}$ (222) at $36.99^{\circ}$. Thus, multiple experimental methods must be utilized to 
conclusively prove or disprove the films as $\mathrm{LiNbO}_{2}$ such as electrical device measurements or chemical composition measurements.

The chemical composition of the deposited films was analyzed using x-ray photoelectron spectroscopy (XPS). In addition to the chemical composition, the oxidation state of the niobium atoms was determined by shifts in the niobium $3 \mathrm{~d}$ signal at a binding energy between $200-215 \mathrm{eV}$. While the chemical composition of niobium and oxygen can be easily determined by XPS, lithium has only a single XPS peak at a binding energy of $54.9 \mathrm{eV}$ which unfortunately overlaps with the niobium $4 \mathrm{~s}$ signal at $55.6 \mathrm{eV}$. This makes quantization of lithium content via XPS difficult.

The oxygen to niobium ratio was determined by dividing the total area under the $\mathrm{O} 1 \mathrm{~s}$ peak by the area under the $\mathrm{Nb} 3 \mathrm{~d}$ peak. Each area was scaled by known relative sensitivity factors for these peaks, which are 2.4 for niobium and 0.66 for oxygen [17]. The niobium oxidation state reported herein is a weighted mean value calculated, according to Equation 1, from fitting the niobium XPS spectra.

Mean Oxidation $=\frac{\sum_{n=0}^{n=5} n * \text { PeakArea }(n)}{\sum_{n=0}^{n=5} \text { PeakArea }(n)}$

Equation 1

where $\mathrm{n}$ is the integer oxidation states of niobium $(0-5)$ used to fit the measured XPS spectrum. These integer oxidation states exhibit doublets in XPS with known binding energies that shift to higher energy as niobium becomes oxidized [18]. All oxygen to niobium ratios and niobium oxidation states reported herein are measured from XPS spectra after argon milling into the film. This procedure avoids sample to sample variations in surface oxide formation, but results in lower reported oxygen to niobium ratios than the actual film composition due to preferential sputtering removing more oxygen than niobium [19].

\section{Results and Discussion}

An initial coarse study of deposition conditions was performed varying the niobium target power, lithium oxide target power, operating pressure, and substrate temperature over a wide range of deposition conditions. Many of these films resulted in $\mathrm{Li}-\mathrm{Nb}-\mathrm{O}$ phases not useful in memristive devices. From this wide variation of deposition conditions, a deposition condition that resulted in $\mathrm{Li}_{x} \mathrm{NbO}_{2-y}$ was observed. This condition became the central deposition condition about which this study's parameters were varied.

\section{Central Deposition Condition}

The central deposition condition found from the coarse optimization was $\mathrm{P}_{\mathrm{Nb}}=50$ $\mathrm{W}, \mathrm{P}_{\mathrm{Li} 2 \mathrm{O}}=200 \mathrm{~W}$, Pressure $=1 \mathrm{mTorr}$, and Temperature $=$ Unheated $\left(\approx 30^{\circ} \mathrm{C}\right)$. These conditions were found to produce crystalline material with a strong XRD peak at $36.585^{\circ}$ 
consistent with (101) oriented $\mathrm{LiNbO}_{2-x}$ and also exhibited memristive hysteresis, as shown in Figure 1, when measured under conditions identical to previous $\mathrm{LiNbO}_{2}$ devices [20]. Thus, this material behaves similar to $\mathrm{LiNbO}_{2}$ produced by $\mathrm{MBE}$ [2] or LPEE [20].

Figure 1: (a) X-ray diffractogram for the initial deposition condition showing a single strong peak at $36.585^{\circ}$ corresponding to (101) oriented lithium niobite. (b) Current - voltage curves measured from the sputter deposited material with Ti/Au contacts. These curves exhibit memristive hysteresis similar to previously reported lithium niobite devices.

The XPS spectra for this central deposition condition are shown in Figure 2 where Figure 2a was measured from the film's surface and Figure $2 b$ was measured after milling into the film. The surface spectra can be fit using only the $\mathrm{Nb}^{3+}$ and $\mathrm{Nb}^{5+}$ oxidation states. The ratio of the areas of these two peaks weighted by the oxidation state yields a mean oxidation state of 4.25 as shown in Figure 2a. In contrast, the existence of a surface oxide and preferential sputtering during the XPS depth profile causes a lower mean oxidation state of 2.92 after milling into the film. Preferential sputtering during the depth profile removes more oxygen than niobium and causes a mixture of many oxidation states [19] as shown in Figure 2b. While this ambiguity in absolute oxidation state determination prevents quantization of the exact oxidation state, comparisons from one sample to another allow relative changes in oxidation state to be determined.

Figure 2: (a) Niobium XPS spectrum measured from the surface of the initial deposition condition film. This spectrum can be fit using only the $3+$ and $5+$ oxidation states. (b) Niobium XPS spectrum after milling into the initial deposition condition film. The data requires a broad range of oxidation states in order to fit the spectrum and has a lower mean oxidation state than the surface measurement due to preferential sputtering of the oxygen during milling.

In order to compare the sputter deposited films to a known $\mathrm{LiNbO}_{2}$ standard, XPS of bulk $\mathrm{LiNbO}_{2}$, grown by liquid phase electro-epitaxy [15], was performed. As shown in Figure 3, the niobium XPS spectrum for the sputter deposited film is nearly identical to the XPS spectrum for the bulk grown material indicating that the sputtered material has a chemical environment around the niobium atoms that is similar to the previously characterized [15] bulk grown material.

Figure 3: XPS spectra for the sputtered initial deposition condition and for bulk grown lithium niobite. These two spectra match closely indicating similar chemical environments around the niobium atom in these two materials. 
In order to investigate the effects of various deposition parameters on the physical and chemical properties of the deposited film, niobium target power, lithium oxide target power, operating pressure, and substrate temperature were varied around the central deposition conditions.

\section{Niobium Power}

The niobium target power was varied between $30 \mathrm{~W}$ and $150 \mathrm{~W}$. Over this range, the thickness of the deposited material increases nearly linearly as the niobium target power is increased at a rate of $5.6 \mathrm{~nm} / \mathrm{W}$, as shown in Figure 4a. In addition, the crystallinity of the film, measured by XRD intensity, increases rapidly for low niobium target powers and decreases slowly for higher niobium power, which may occur due to chemical changes in the film's deposited with more niobium. Chemically, as shown in Figure $4 \mathrm{~b}$, the niobium oxidation state decreases as the niobium target power increases. This is consistent with adding more niobium to the film and making the film more metal rich. The oxygen to niobium ratio also decreases as the niobium target power increases, although the film deposited with $40 \mathrm{~W}$ niobium power is an outlier from this trend. These chemical trends indicate a narrow niobium power range of $40-50 \mathrm{~W}$ wherein the desired $\mathrm{Li}_{x} \mathrm{NbO}_{2}$ films are obtained. This region also corresponds with the best XRD figures of merit as shown in figure $4 \mathrm{a}$.

Figure 4: (a) Physical trends in changing niobium target power showing an increase in film thickness as the niobium target power increases and a peak in crystallinity as measured by XRD intensity. (b) Chemical trends in changing niobium target power showing a generally decreasing trend in both niobium oxidation state and oxygen to niobium ratio as the niobium target power increases.

\section{Lithium Oxide Power}

The lithium oxide $\left(\mathrm{Li}_{2} \mathrm{O}\right)$ target power was varied between $100 \mathrm{~W}$ and $300 \mathrm{~W}$. Over this range, the thickness of the deposited material increases as the $\mathrm{Li}_{2} \mathrm{O}$ target power is increased in a nearly linear manner at a rate of $1.55 \mathrm{~nm} / \mathrm{W}$, as shown in Figure 5a. Unlike the range of niobium target powers investigated, the film crystallinity decreases throughout the entire range of $\mathrm{Li}_{2} \mathrm{O}$ target power. Not surprisingly, increasing the $\mathrm{Li}_{2} \mathrm{O}$ target power increases the oxidation state in the deposited film. This is consistent with adding more oxygen to the film. However, the measured oxygen to niobium ratio does not track with the niobium oxidation state and is mostly unchanged by changing the $\mathrm{Li}_{2} \mathrm{O}$ target power.

Figure 5: (a) Physical trends in changing $\mathrm{Li}_{2} \mathrm{O}$ target power showing an increase in film thickness as the $\mathrm{Li}_{2} \mathrm{O}$ target power increases. Unlike the range of niobium target powers investigated, the film crystallinity decreases over the range of $\mathrm{Li}_{2} \mathrm{O}$ target powers investigated. (b) Chemical trends 
in changing $\mathrm{Li}_{2} \mathrm{O}$ target power showing a generally increasing trend in niobium oxidation state and mostly constant oxygen to niobium ratio as the $\mathrm{Li}_{2} \mathrm{O}$ target power increases.

\section{Pressure}

The operating pressure was varied between 1 mTorr and 30 mTorr. Over this pressure range the mean free path of an atom should vary between $20 \mathrm{~cm}$ and $0.7 \mathrm{~cm}$ respectively according to the kinetic model for gas collisions shown in Equation 2.

$\Gamma=\frac{k_{B} T}{\pi d^{2} P}$

Equation 2

where $\Gamma$ is the mean free path, $k_{B}$ is Boltzmann's constant, $T$ is temperature, $d$ is the diameter of an atom (Argon assumed), and $P$ is pressure. The range of mean free paths investigated extends both longer and shorter than the $10 \mathrm{~cm}$ target to substrate distance spanning cases from molecular to viscous flow which result in dramatically different energetic species impinging on the substrates.

Increasing the pressure between 1 - 10 mTorr had little impact on the thickness of the deposited film, although the film crystallinity decreased over this range, as shown in Figure 6a. The decrease in film crystallinity may be due to increased scattering as the mean free path becomes shorter than the target to substrate distance. In sputter deposition, unscattered atoms carry a large amount of kinetic energy, typically $4-40$ $\mathrm{eV}$ [21], which can provide the energy necessary for crystallization. The films deposited at pressures between $1-10$ mTorr show only small changes to the niobium oxidation state and oxygen to niobium ratio.

However, the film deposited at 30 mTorr is approximately $1 / 3$ the thickness of the films deposited at lower pressures and exhibits an oxygen to niobium ratio approximately 2.5 times higher than the films deposited at lower pressure despite a relatively unchanging niobium oxidation state. Since the oxygen to niobium ratio increased without increasing the niobium oxidation state, excess lithium must be incorporated into the film and while we cannot measure Li-O bonding with XPS, such bonding must exist. The film deposited at 30 mTorr also exhibits a higher crystallinity than the low pressure trend would predict which may be due to the change in chemical composition.

Figure 6: (a) Physical trends in changing pressure showing a relatively unchanging film thickness between 1-10 mTorr with decreasing crystallinity and a film approximately $1 / 3$ the thickness at 30 mTorr (b) Chemical trends in changing pressure showing small increases in the niobium oxidation state and oxygen to niobium ratio at pressures between 1-10 mTorr and a substantially higher oxygen to niobium ratio at $30 \mathrm{mTorr}$.

\section{Substrate Temperature}


The substrate temperature was varied between room temperature and $800^{\circ} \mathrm{C}$. Unintentional heating of the substrate at room temperature was controlled by water cooling the platen. The measured temperature did not exceed $40^{\circ} \mathrm{C}$ for the unintentionally heated films. As shown in Figure 7, increasing the substrate temperature to $500{ }^{\circ} \mathrm{C}$ had little effect on the film thickness, crystallinity, niobium oxidation state, or oxygen to niobium ratio. However, increasing the substrate temperature to $800{ }^{\circ} \mathrm{C}$ caused a decrease in the film thickness and crystallinity. The film deposited at $800{ }^{\circ} \mathrm{C}$ also exhibited a higher niobium oxidation state and higher oxygen to niobium ratio. In addition to the quantitative chemical measurements, the film deposited at $800{ }^{\circ} \mathrm{C}$ was transparent as opposed to the opaque films deposited at lower temperatures. The XRD peak for the $800{ }^{\circ} \mathrm{C}$ sample was also located at a slightly higher angle $\left(2 \theta-\omega=36.87^{\circ}\right)$ than all other films investigated in this study $\left(2 \theta-\omega=36.39^{\circ}-36.76^{\circ}\right)$. Of the known Li$\mathrm{Nb}-\mathrm{O}$ compounds with XRD peaks near $2 \theta-\omega=36^{\circ}-37^{\circ}, \mathrm{Li}_{3} \mathrm{NbO}_{4}$ and $\mathrm{LiNb}_{3} \mathrm{O}_{8}$ are the only transparent compounds, with a band gap of approximately $4 \mathrm{eV}[22,23]$, having a higher ideal niobium oxidation state of $\mathrm{Nb}^{5+}$, and a higher angle XRD peak than all other compounds in this range. Thus, the $800{ }^{\circ} \mathrm{C}$ sample is likely dominated by $\mathrm{Li}_{3} \mathrm{NbO}_{4}$ or $\mathrm{LiNb}_{3} \mathrm{O}_{8}$.

Figure 7: (a) Physical trends in changing substrate temperature showing a small decrease in film thickness and crystallinity between $30-500{ }^{\circ} \mathrm{C}$ and a larger decrease in both film thickness and crystallinity at $800{ }^{\circ} \mathrm{C}$ (b) Chemical trends in changing substrate temperatures showing small increases in the niobium oxidation state and oxygen to niobium ratio between $30-500{ }^{\circ} \mathrm{C}$ and a larger increase in niobium oxidation state and oxygen to niobium ratio at $800{ }^{\circ} \mathrm{C}$.

\section{Excess Lithium}

It may be noted that no linear combination of $\mathrm{Li}_{2} \mathrm{O}$ and $\mathrm{Nb}$ can form $\mathrm{LiNbO}_{2}$. In order to obtain the ideal 2:1 oxygen to niobium ratio, excess lithium must a) not incorporate into the deposited film or b) evolve out of the film after it has been deposited. All of the films examined herein, with the exception of the film deposited at $800{ }^{\circ} \mathrm{C}$, reacted with air upon removal from the sputtering chamber to form an insulating film on top of the deposited film, as shown in Figure 8. Examining the XPS spectra for lithium, niobium, and oxygen, shown in Figure 9, the surface layer is composed of lithium and oxygen with no niobium present. After milling into the deposited film, niobium and oxygen are both present. Lithium may also be present after milling, but the lithium 1s spectrum overlaps the niobium 4s spectrum making its quantization indeterminate. It can also be noted from the XPS spectra in Figure 9 that the oxygen binding energy shifts from the surface region to the bulk of the film, which indicates that the oxygen is bound differently on the surface and after milling. This suggests two distinctly different films as shown in the cross sectional SEM in Figure 8. The surface 
layer that forms is water soluble and upon dissolution turns deionized water basic, $\mathrm{pH}$ of $10-11$ in $100 \mathrm{~mL}$ of water, likely due to the formation of $\mathrm{LiOH}$.

Figure 8: Cross sectional SEM of a sputter deposited film showing a layer on top of the deposited film. This layer forms after deposition due to a reaction with the air and is water soluble.

Figure 9: $(\mathrm{a}-\mathrm{c})$ XPS spectra for lithium, niobium, and oxygen of the reacted surface layer and after milling through to the main deposited film. The surface layer is composed of lithium and oxygen with no niobium present. The main film contains at least niobium and oxygen, but the lithium XPS signal is washed out by the niobium $4 \mathrm{~s}$ signal.

\section{Conclusion}

We have demonstrated the room temperature deposition of thin film crystalline lithium niobite $\left(\mathrm{LiNbO}_{2}\right)$ with a (101) orientation by co-sputtering from a lithium oxide $\left(\mathrm{Li}_{2} \mathrm{O}\right)$ target and a niobium target along with a systematic method to optimize its deposition. This material displays memristive hysteresis and exhibits XPS spectra similar to MBE and bulk grown $\mathrm{LiNbO}_{2}$. The effects of various deposition parameters were investigated including niobium target power, lithium oxide target power, operating pressure, and substrate pressure. Maintaining low pressure and temperature improved the deposition rate and film crystallinity without significantly impacting the film's chemical properties. Increasing the niobium or lithium oxide target powers resulted in increased deposition rates. However, changing the target powers also changed the chemical composition of the deposited film resulting in a narrow window in which memristive $\mathrm{LiNbO}_{2}$ can be achieved. Thus, sputter deposition can be tailored to result in $\mathrm{LiNbO}_{2}$ useful in memristive applications even for near ambient conditions, potentially useful in hybrid integration of memristors with other computational or optical systems.

\section{Acknowledgements}

This work was supported by the Defense Threat Reduction Agency, Basic Research Award HDTRA-1-12-1-0031 administered by Dr. James Reed and Dr. Jacob Calkins.

\section{References}

[1] N. Kumada, S. Muramatu, F. Muto, N. Kinomura, S. Kikkawa, M. Koizumi, Topochemical reactions of $\mathrm{Li}_{x} \mathrm{NbO}_{2}$, Journal of Solid State Chemistry, 73 (1988) 33-39.

[2] J.D. Greenlee, W. Calley, W. Henderson, W.A. Doolittle, Halide based MBE of crystalline metals and oxides, Physica status solidi (c), 9 (2012) 155-160.

[3] G. Meyer, R. Hoppe, The First Oxoniobate (III) $\mathrm{LiNbO}_{2}$, Angewandte Chemie International Edition in English, 13 (1974) 744-745. 
[4] M.J. Geselbracht, A.M. Stacy, A.R. Garcia, B.G. Silbernagel, G.H. Kwei, Local environment and lithium ion mobility in lithium niobate $\left(\mathrm{LiNbO}_{2}\right)$ : inferences from structure, physical properties, and NMR, The Journal of Physical Chemistry, 97 (1993) 7102-7107.

[5] A. McDowell, D. Snyderman, M.S. Conradi, B. Silbernagel, A. Stacy, Cross relaxation and atomic motion in $\mathrm{LiNbO}_{2}$, Physical Review B, 50 (1994) 15764.

[6] D. Novikov, V. Gubanov, V. Zubkov, A. Freeman, Electronic structure and electron-phonon interactions in layered $\mathrm{Li}_{x} \mathrm{NbO}_{2}$ and $\mathrm{Na}_{x} \mathrm{NbO}_{2}$, Physical Review $\mathrm{B}, 49$ (1994) 15830.

[7] E. Moshopoulou, P. Bordet, J. Capponi, Superstructure and superconductivity in $\mathrm{Li}_{1-\mathrm{x}} \mathrm{NbO}_{2}(\mathrm{x} \approx 0.7)$ single crystals, Physical Review B, 59 (1999) 9590.

[8] V. Cherkashenko, M. Korotin, V. Anisimov, V. Shumilov, V. Galakhov, D. Kellerman, V. Zubkov, E. Kurmaev, X-ray spectra and electronic structure of $\mathrm{Li}_{x} \mathrm{NbO}_{2}$ superconductor and other niobium oxide compounds, Zeitschrift für Physik B Condensed Matter, 93 (1994) 417-424.

[9] E.R. Ylvisaker, W.E. Pickett, First-principles study of the electronic and vibrational properties of $\mathrm{LiNbO}_{2}$, Physical Review B, 74 (2006) 075104.

[10] J.D. Greenlee, C.F. Petersburg, W. Laws Calley, C. Jaye, D.A. Fischer, F.M. Alamgir, W. Alan Doolittle, In-situ oxygen $x$-ray absorption spectroscopy investigation of the resistance modulation mechanism in $\mathrm{LiNbO}_{2}$ memristors, Applied Physics Letters, 100 (2012) 182106-182106-182104.

[11] J.C. Shank, M.B. Tellekamp, W.A. Doolittle, Evidence of ion intercalation mediated band structure modification and opto-ionic coupling in lithium niobite, Journal of Applied Physics, 117 (2015) 035704.

[12] J.D. Greenlee, W. Calley, M.W. Moseley, W.A. Doolittle, Comparison of Interfacial and Bulk lonic Motion in Analog Memristors, IEEE Transactions on Electron Devices, 60 (2013) 427-432.

[13] J.D. Greenlee, J.C. Shank, M.B. Tellekamp, W.A. Doolittle, Spatiotemporal drift-diffusion simulations of analog ionic memristors, Journal of Applied Physics, 114 (2013) 034504.

[14] M.J. Geselbracht, T.J. Richardson, A.M. Stacy, Superconductivity in the layered compound $\mathrm{Li}_{x} \mathrm{NbO}_{2}$, Nature, 345 (1990) 324 - 326.

[15] J.D. Greenlee, J.C. Shank, M.B. Tellekamp, B. Gunning, C.A. Fabien, A. Doolittle, Liquid Phase ElectroEpitaxy of Memristive LiNbO ${ }_{2}$ Crystals, Crystal Growth \& Design, 14 (2014) 2218-2222.

[16] W.E. Henderson, W. Laws Calley, A.G. Carver, H. Chen, W. Alan Doolittle, A versatile metal-halide vapor chemistry for the epitaxial growth of metallic, insulating and semiconducting films, Journal of Crystal Growth, 324 (2011) 134-141.

[17] C. Wagner, L. Davis, M. Zeller, J. Taylor, R. Raymond, L. Gale, Empirical atomic sensitivity factors for quantitative analysis by electron spectroscopy for chemical analysis, Surface and interface analysis, 3 (1981) 211-225.

[18] C.F. Miller, G.W. Simmons, R.P. Wei, High temperature oxidation of $\mathrm{Nb}, \mathrm{NbC}$ and $\mathrm{Ni}_{3} \mathrm{Nb}$ and oxygen enhanced crack growth, Scripta Materialia, 42 (2000) 227-232.

[19] P. Karulkar, Effects of sputtering on the surface composition of niobium oxides, Journal of Vacuum Science \& Technology, 18 (1981) 169-174.

[20] J.D. Greenlee, J.C. Shank, M.B. Tellekamp, E.X. Zhang, J. Bi, D.M. Fleetwood, M.L. Alles, R.D. Schrimpf, W.A. Doolittle, Radiation Effects on $\mathrm{LiNbO}_{2}$ Memristors for Neuromorphic Computing Applications, IEEE Trans. Nucl. Sci., 60 (2013) 4555-4562.

[21] J.A. Thornton, Influence of apparatus geometry and deposition conditions on the structure and topography of thick sputtered coatings, Journal of Vacuum Science \& Technology, 11 (1974) 666-670.

[22] Y.-J. Hsiao, T.-H. Fang, S.-J. Lin, J.-M. Shieh, L.-W. Ji, Preparation and luminescent characteristic of $\mathrm{Li}_{3} \mathrm{NbO}_{4}$ nanophosphor, Journal of Luminescence, 130 (2010) 1863-1865.

[23] G. Blasse, Luminescence of lithium niobate $\left(\mathrm{LiNb}_{3} \mathrm{O}_{8}\right)$, physica status solidi (a), 20 (1973) K99-K102. 
a)

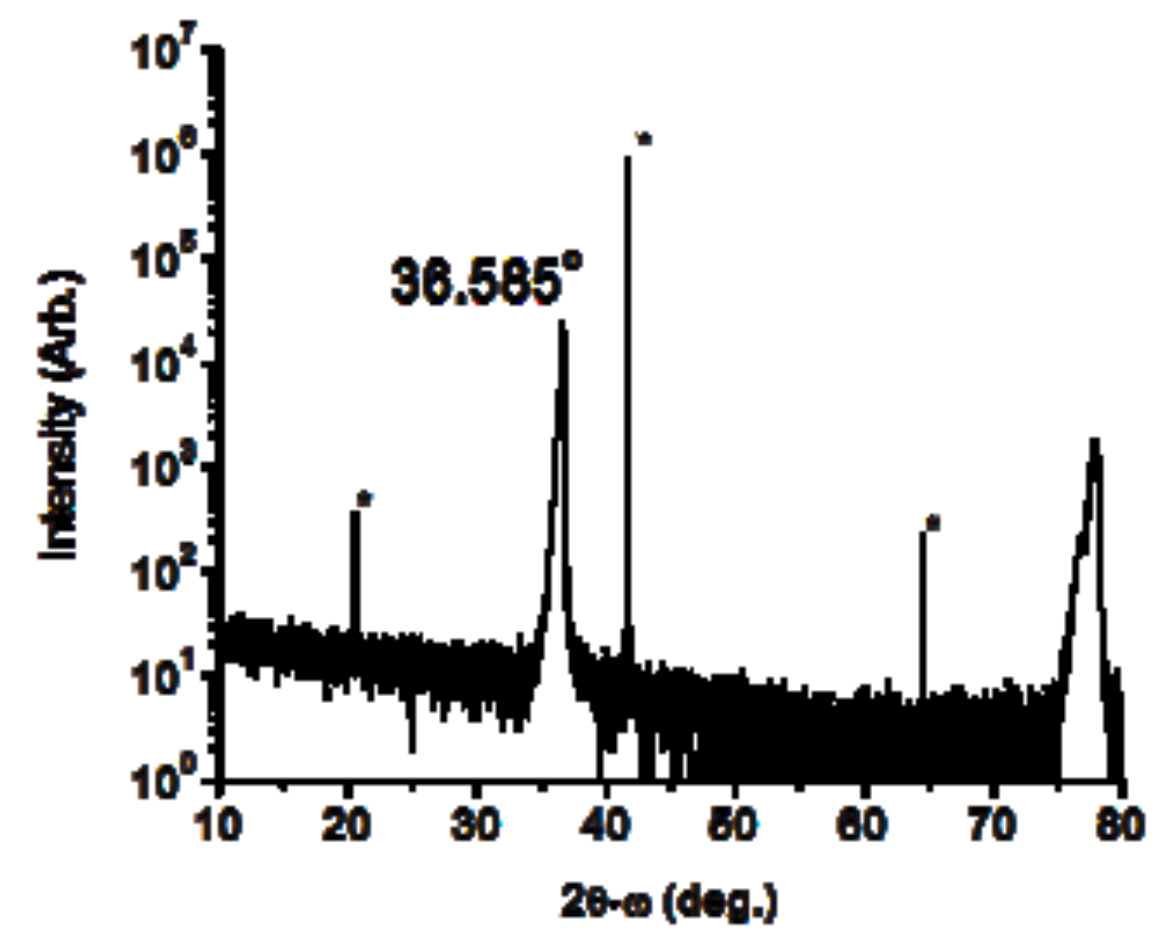

b)

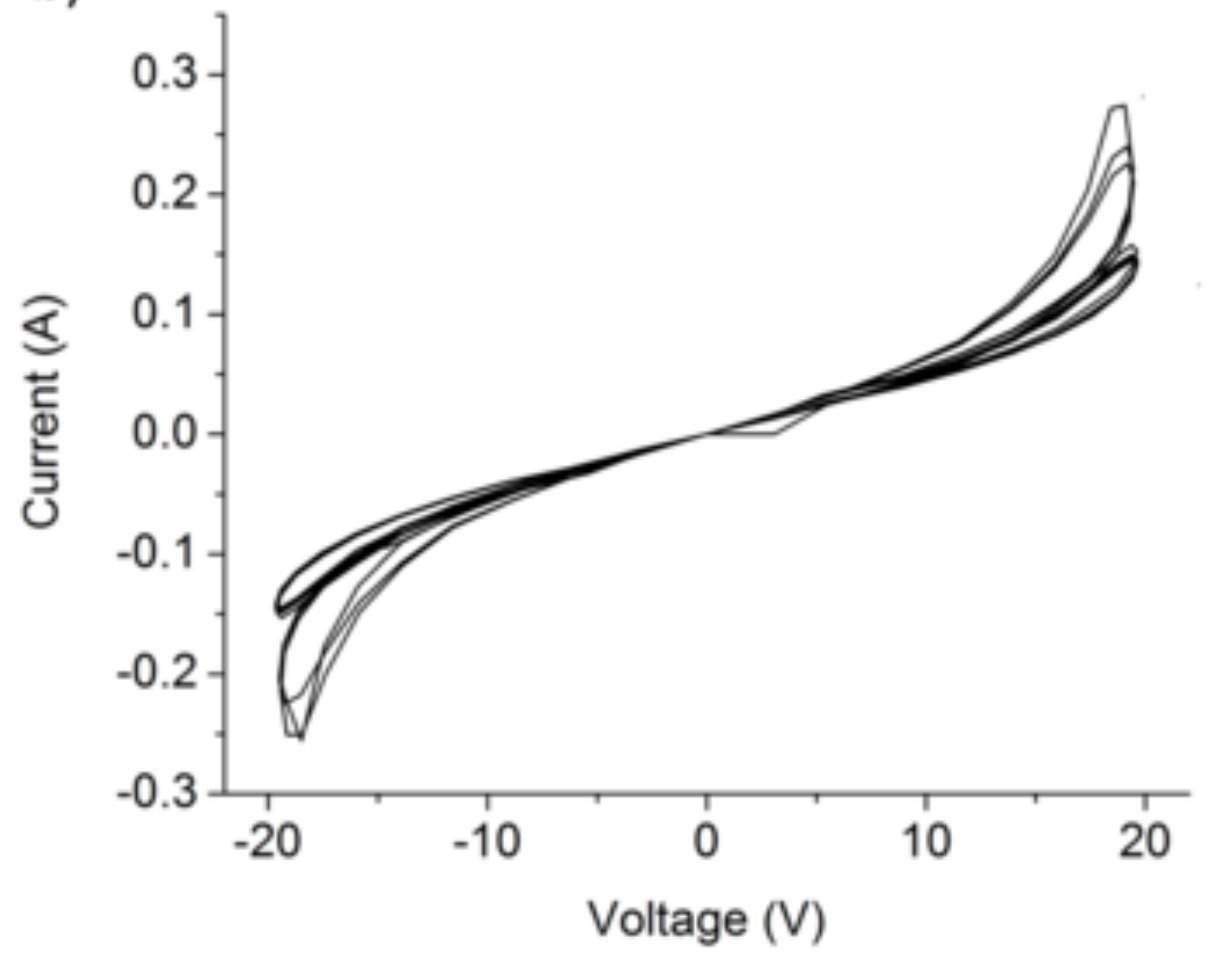


a)

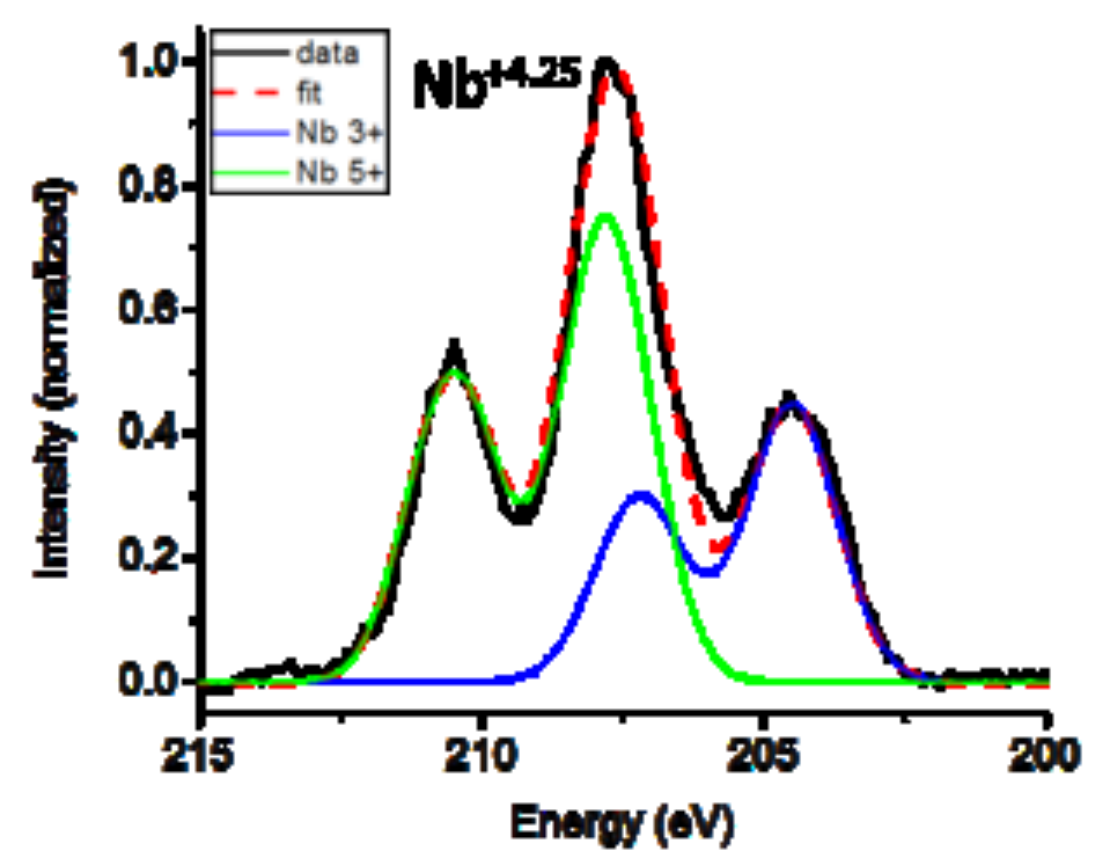

b)

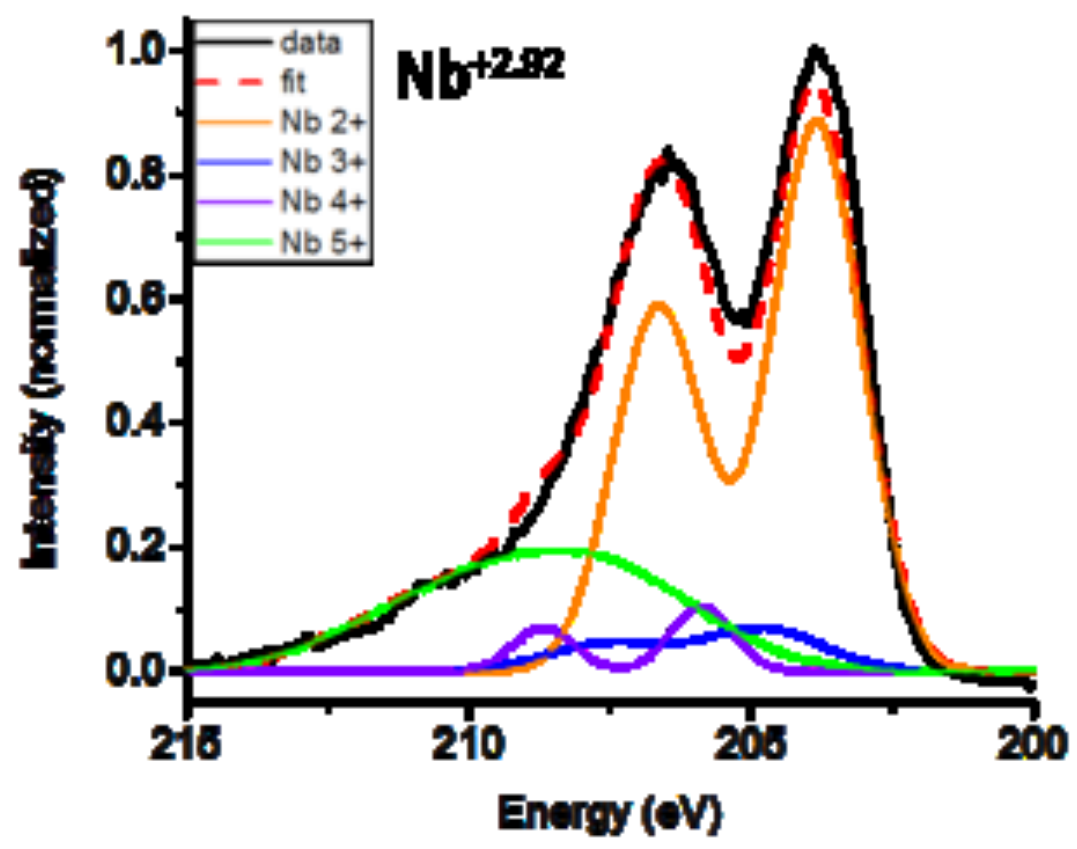




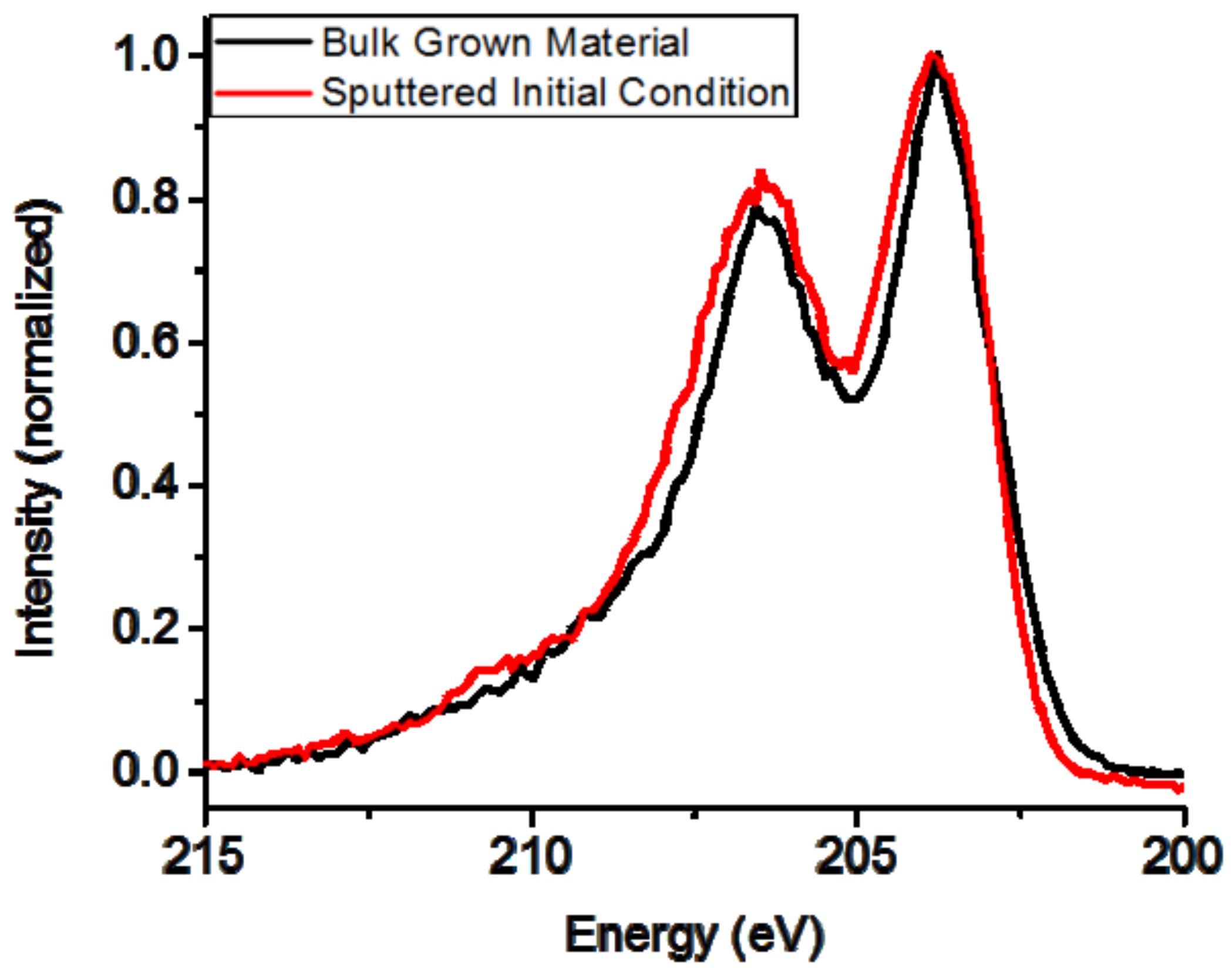


a)

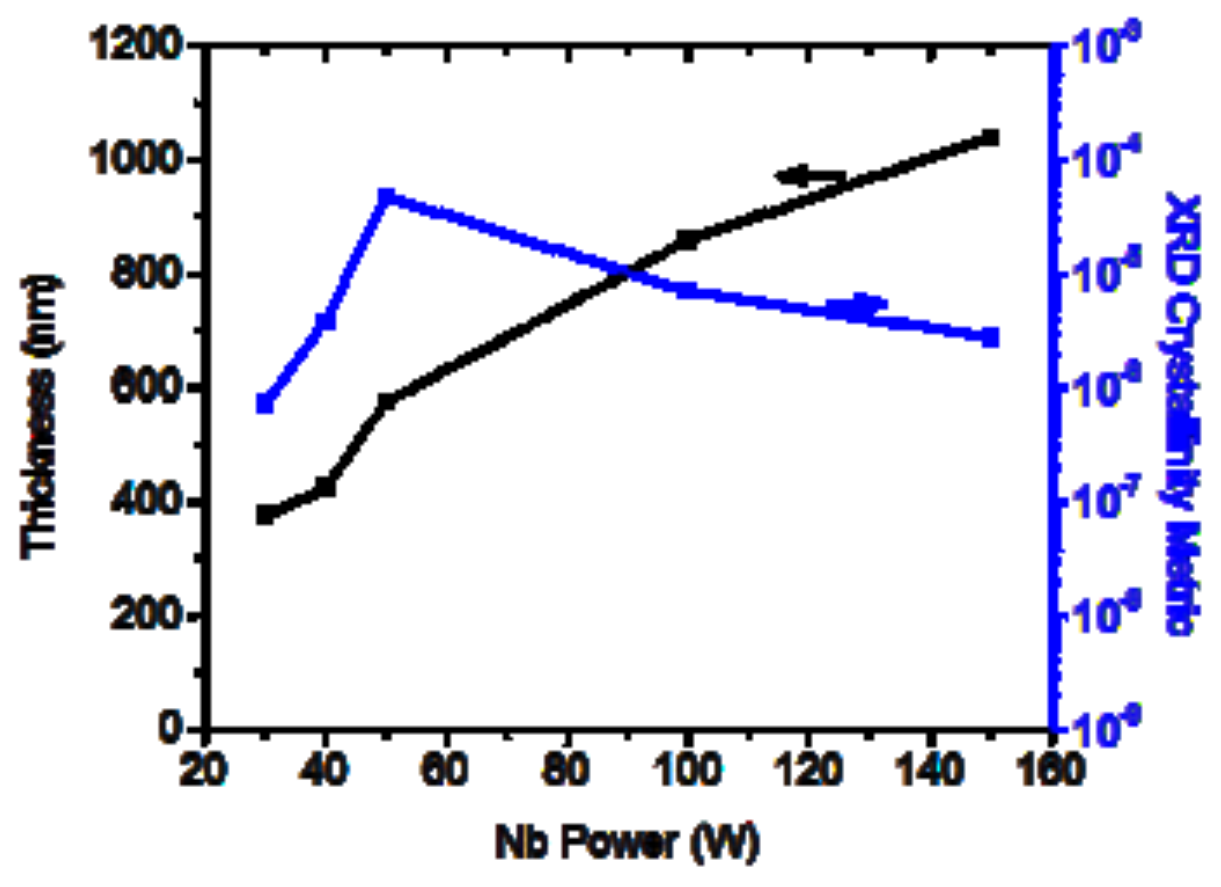

b)

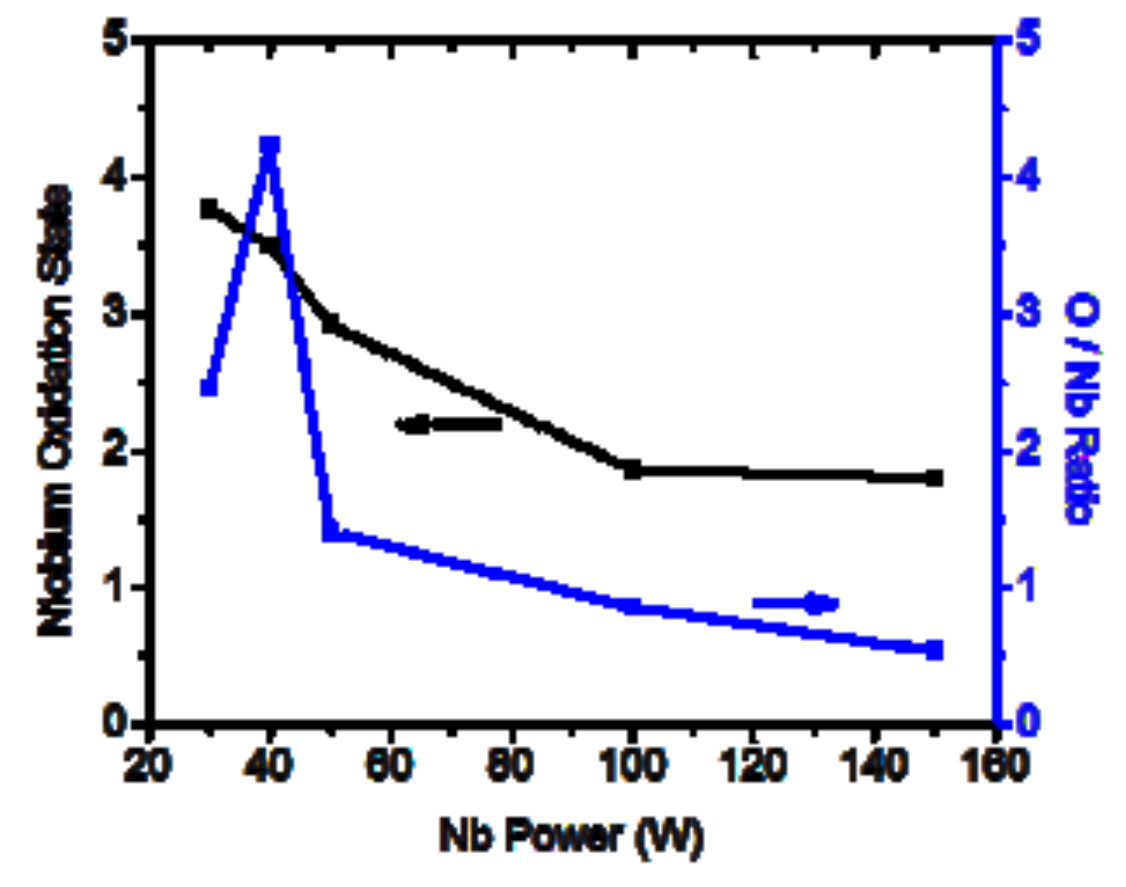


a)

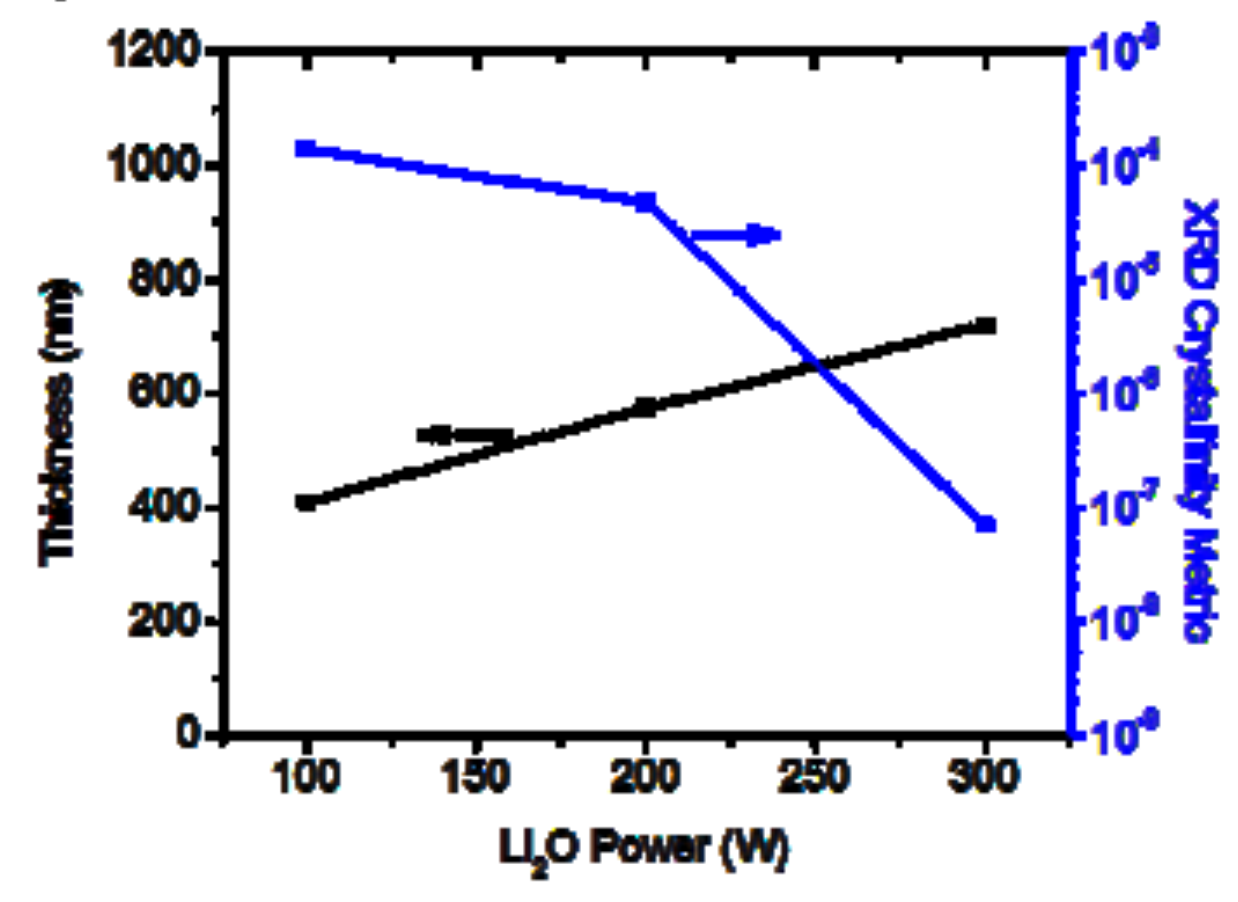

b)

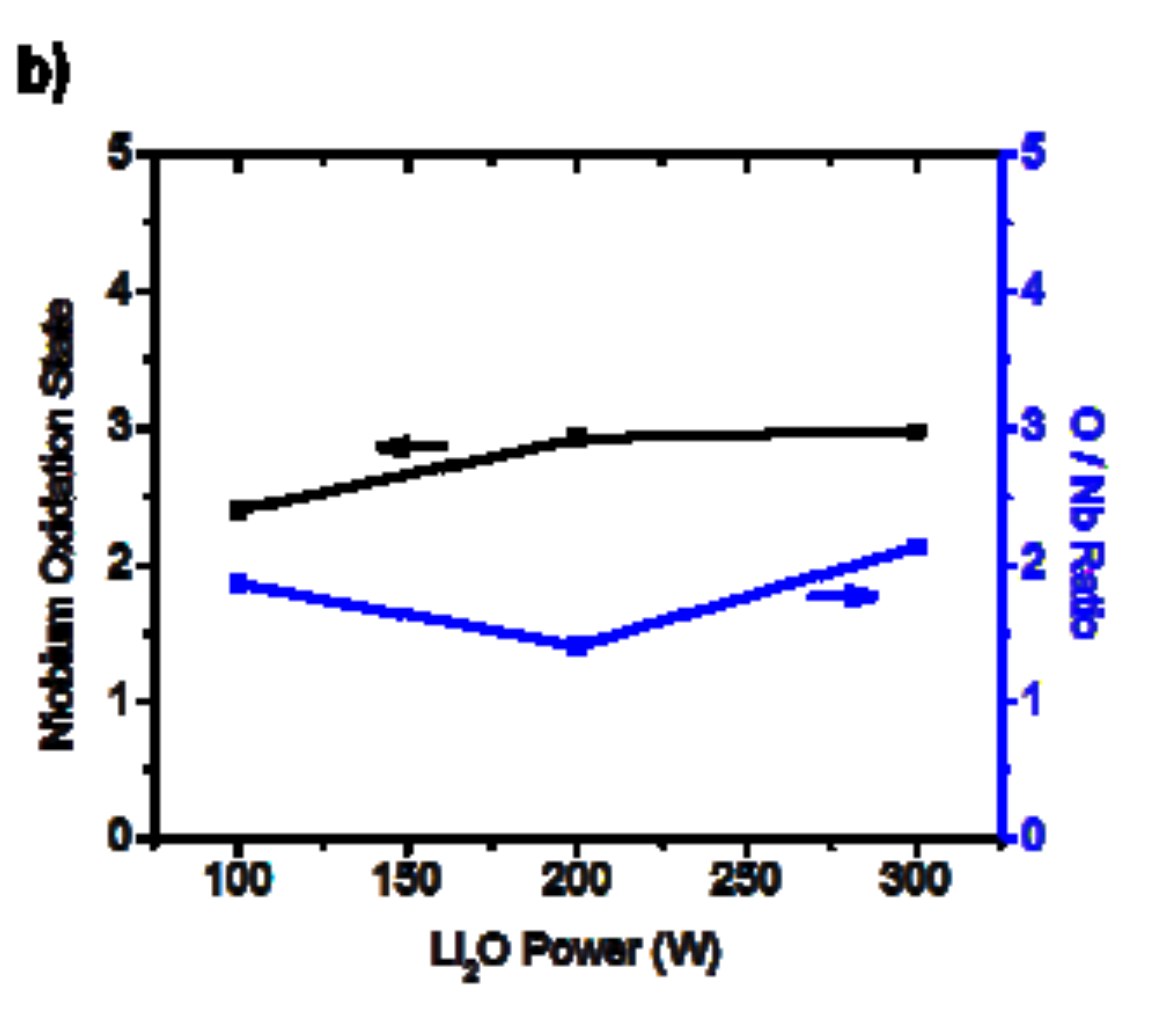

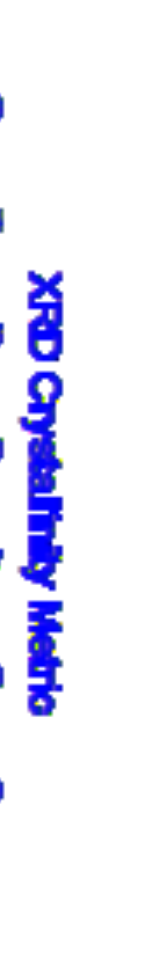


a)

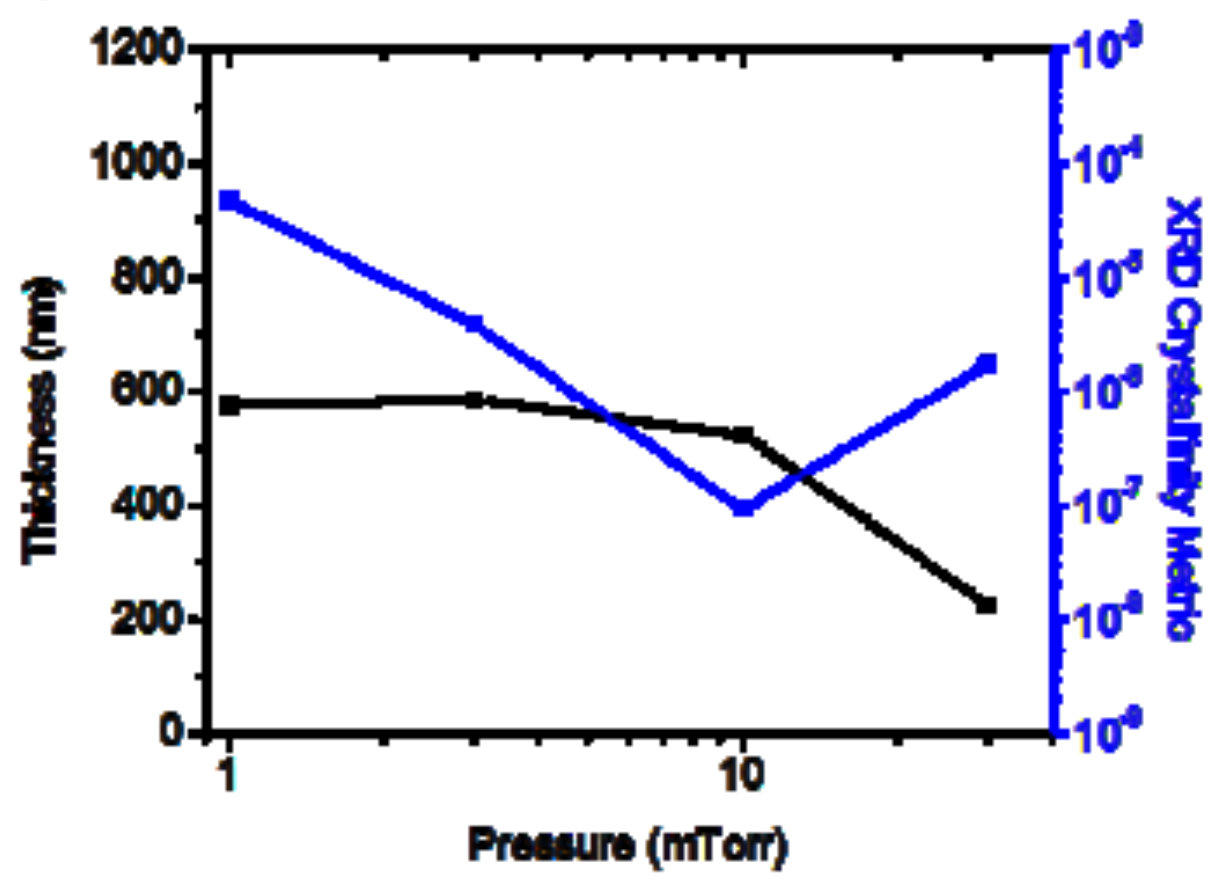

b)

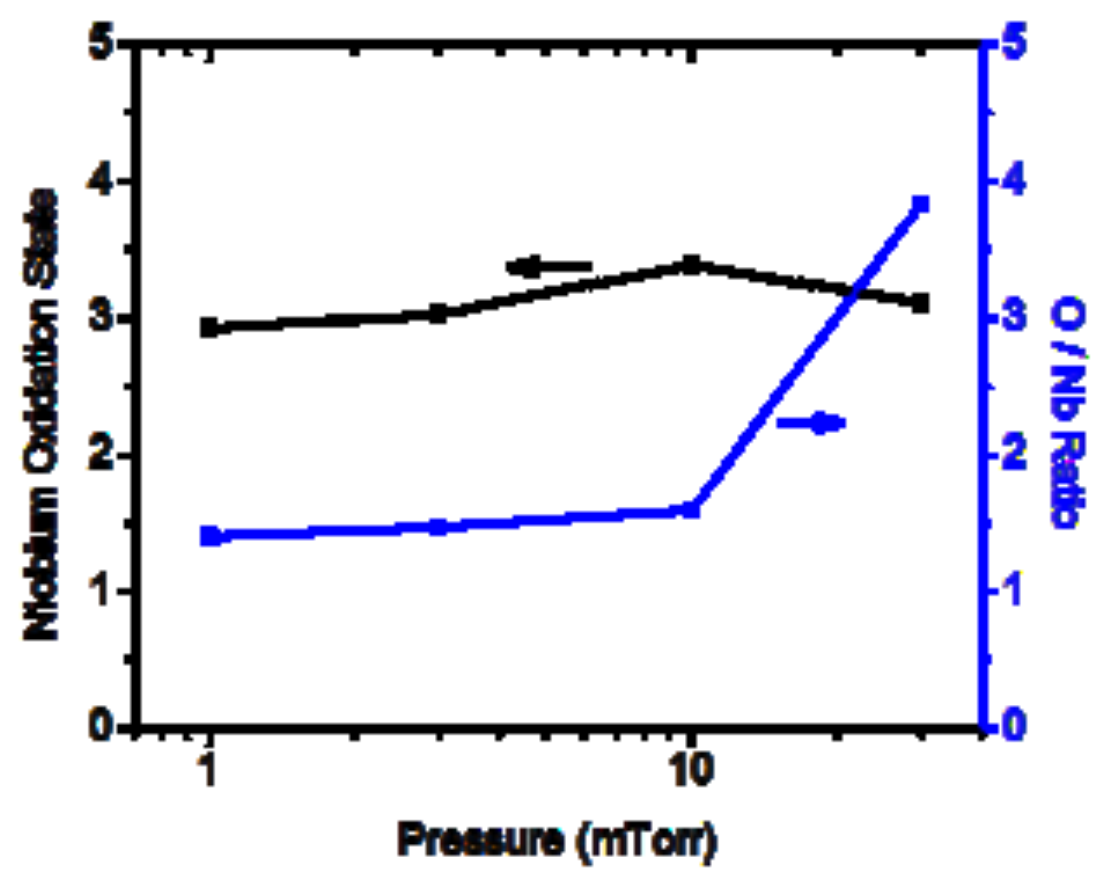


a)

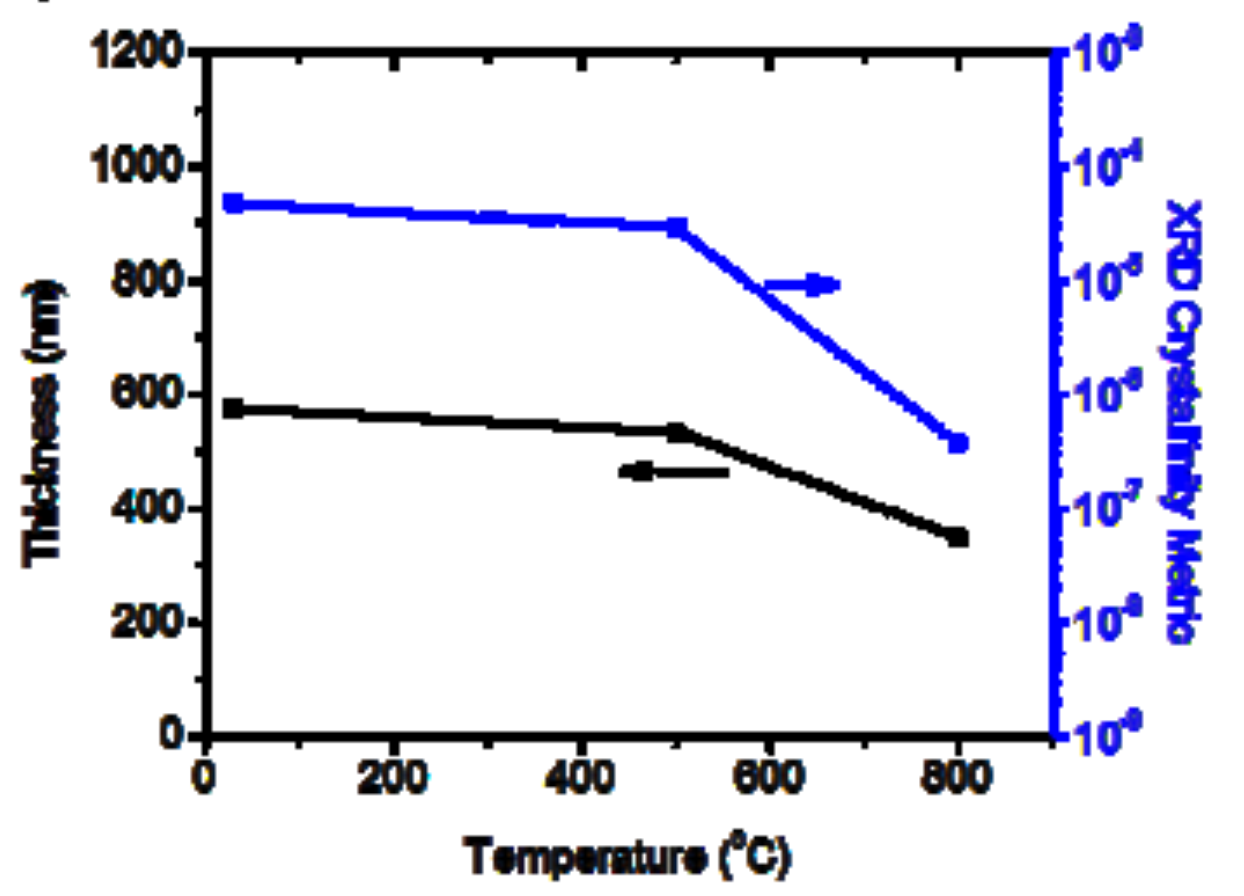

b)

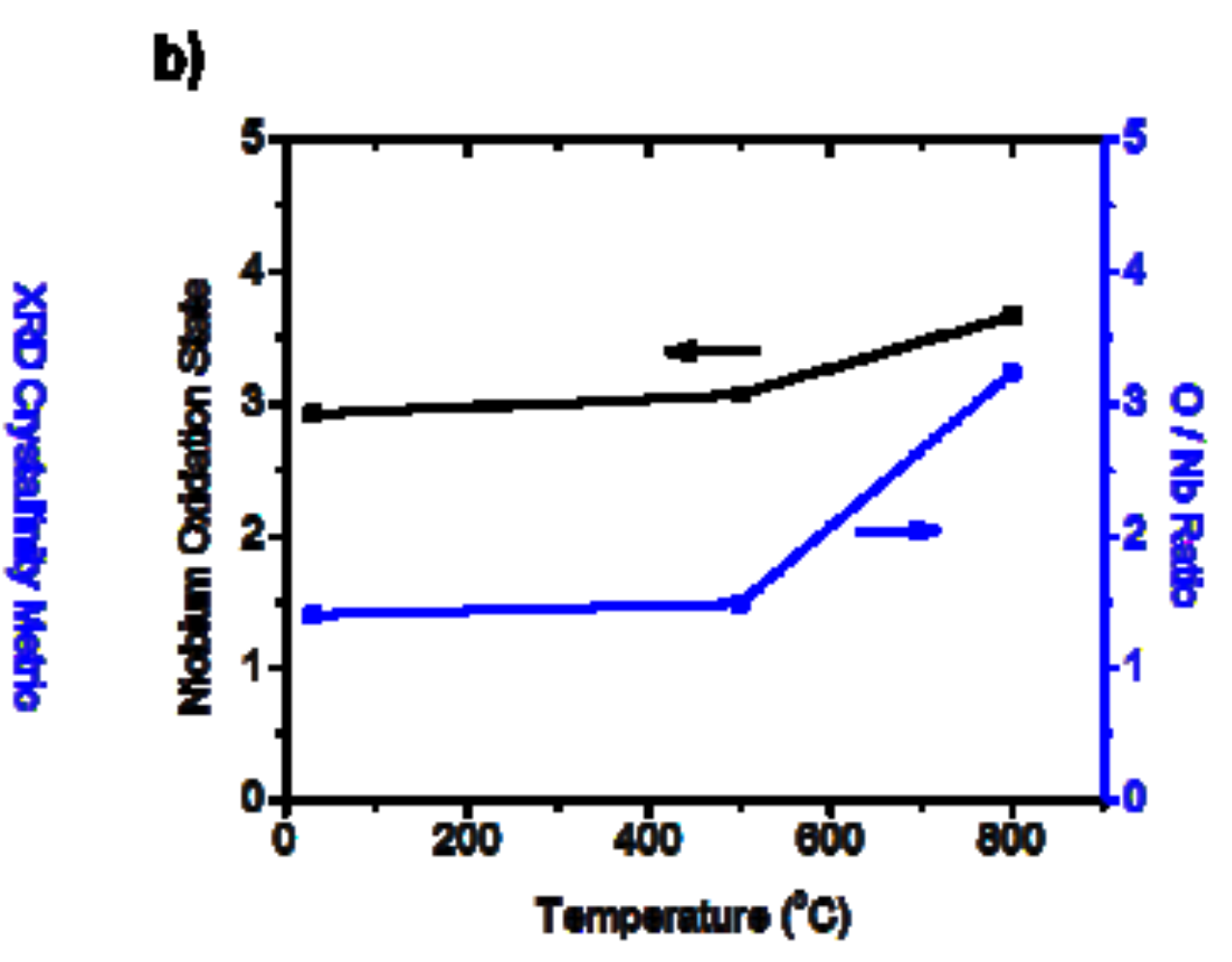



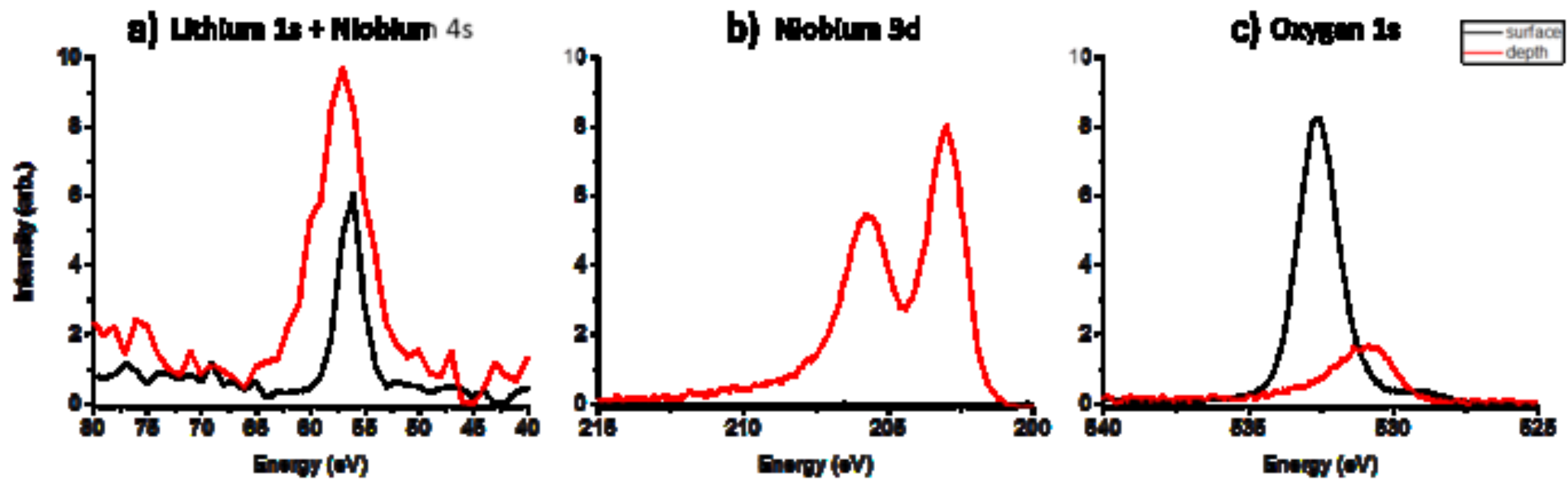